\title{
SPECIAL PROPERTIES OF MEASURE PRESERVING TRANSFORMATIONS
}

\author{
STEPHEN P. DILIBERTO
}

1. Summary. In studying problems ${ }^{1}$ concerned with the qualitative description of bounded trajectories, in a region free of singular points, associated with a flow

$$
d x_{i} / d t=P_{i}\left(x_{1}, \cdots, x_{n}\right) \quad(i=1, \cdots, n)
$$

the $P_{i}$ being holomorphic in the $x_{i}$, we considered the possibility of finding "point conditions" on the $P_{i}$ which would insure a smooth behavior on the part of a trajectory-or more specifically, on any motion in its limit set. For example, the restriction that the transformation defined by equations (1.1) preserve $n$ measure is expressible by the point condition

$$
\sum_{i=1}^{n} \frac{\partial P_{i}}{\partial x_{i}}=0
$$

Being unable to ensure the behavior desired by this condition, we sought stronger conditions. In particular we asked, "What are the conditions on the $P_{i}$ such that the transformations defined by (1.1) preserves $p$ measure where $p$ is restricted to values $1 \leqq p \leqq n-1$ ?" The answer to this question and also to the question, "Is this a restrictive condition?" is contained in the following theorem.

THEOREM I. The condition that the flow defined by

$$
d x_{i} / d t=P_{i}\left(x_{1}, \cdots, x_{n}\right) \quad(i=1, \cdots, n),
$$

the $P_{i}$ being holomorphic, preserve $p$ measure, $p$ any (fixed) integer between 1 and $n-1$, is that

$$
\partial P_{i} / \partial x_{j}=-\partial P_{j} / \partial x_{i}
$$

for all $i$ and $j$. These conditions imply that the motion is rigid.

It is an open and apparently difficult question as to whether every point transformation (we are considering only homeomorphisms)of a sufficiently differentiable class-of $E^{3}$ onto itself is obtainable from a flow, that is, from the solutions of a system of first order equations of the type (1.1). (In $E^{2}$ there are point transformations which are not embeddable in flows.) Thus it is natural to ask for how

Presented to the Society, April 17, 1948; received by the editors April 16, 1948.

${ }^{1}$ On systems of ordinary differential equations, which will appear elsewhere. 
large a class of transformations the above result holds. We have a partial sharpening of Theorem $I$ to the following theorem.

TheOREM II. The only point transformations, of class $C^{3}$, of Euclidean three space into itself which preserve area are rigid motions (and reflections).

2. A determinant expansion. We state next, without proof, a well known property about the product of an $n \times p$ by $p \times n$ matrix:

LEMMA. Let

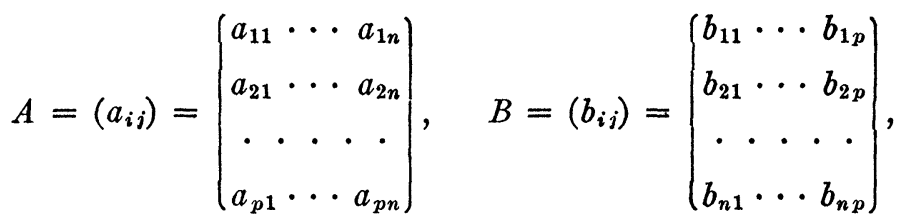

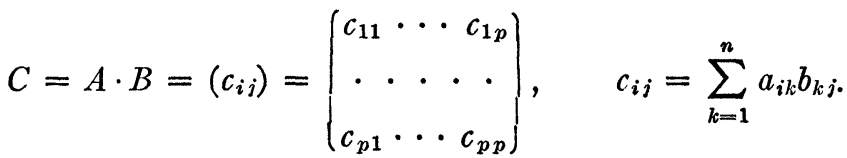

Then

$$
|C|=\sum_{\left(l_{i}\right)}\left|\begin{array}{ccc}
a_{1 l_{1}} & \cdots & a_{1 l_{p}} \\
\cdot & \cdot & \cdot \\
a_{p l_{1}} & \cdots & a_{p l_{p}}
\end{array}\right|\left|\begin{array}{ccc}
b_{l_{1} 1} & \cdots & b_{l_{1} p} \\
\cdot & \cdot & \cdot \\
b_{l_{p} 1} & \cdots & b_{l_{p} p}
\end{array}\right|
$$

This lemma says that the determinant of the product of $p \times n$ matrix by an $n \times p$ matrix is expressible as the sum of products of $p$ square matrices, the first factor being a $p$ square determinant from $A$ selected by choosing columns $l_{1}, \cdots, l_{p}$ and the second factor being a $p$ square determinant from $B$ selected by choosing rows $l_{1}, \cdots, l_{p}$ from $B$, the summation being over all ways, $C_{n, p}$, of choosing $p$ numbers from a set of $n$ (and we take $l_{1}<l_{2}<\ldots<l_{p}$ ).

We make use of this lemma to generalize the well known product formula for Jacobians. Let $X_{i}$ and $x_{i}$ be functions of class $C^{2}$ where

$$
\begin{aligned}
X_{i} & =X_{i}\left(x_{1}, \cdots, x_{n}\right), & i & =1, \cdots, p, \\
x_{i} & =x_{i}\left(u_{1}, \cdots, u_{p}\right), & i & =1, \cdots, n ;
\end{aligned}
$$

then, the Jacobian of the $X_{i}$ with respect to the $u_{i}$ is expressible as

$$
\frac{\partial\left(X_{1}, \cdots, X_{p}\right)}{\partial\left(u_{1}, \cdots, u_{p}\right)}=\left|\frac{\partial X_{i}}{\partial u_{j}}\right|=\left|\begin{array}{c}
\partial X_{1} / \partial u_{1} \cdots \partial X_{1} / \partial u_{n} \\
\cdot . \cdot . \cdot . \cdot . \\
\partial X_{n} / \partial u_{1} \cdots \partial X_{n} / \partial u_{n}
\end{array}\right|
$$


but

$$
\partial X_{i} / \partial u_{j}=\sum_{k=1}^{n}\left(\partial X_{i} / \partial x_{k}\right) \cdot\left(\partial x_{k} / \partial u_{j}\right)
$$

and so $\partial\left(X_{1}, \cdots, X_{p}\right) / \partial\left(u_{1}, \cdots, u_{p}\right)$ has the same form as $|C|$ in the last lemma. Thus

$$
\frac{\partial\left(X_{1}, \cdots, X_{p}\right)}{\partial\left(u_{1}, \cdots, u_{p}\right)}=\sum_{\left(l_{i}\right)} \frac{\partial\left(X_{1}, \cdots, X_{p}\right)}{\partial\left(u_{l_{1}}, \cdots, u_{l_{p}}\right)} \cdot \frac{\partial\left(x_{l_{1}}, \cdots, x_{l_{p}}\right)}{\partial\left(u_{1}, \cdots, u_{p}\right)} .
$$

3. Proof of Theorem I. Beginning with equation (1.1), let

$$
x_{i}=f_{i}\left(x_{10}, \cdots, x_{n 0}, t\right)
$$

be the solution of that equation which at time $t_{0}$ passes through the point $\left(x_{10}, \cdots, x_{n 0}\right)$. We take a regular surface to be given by

$$
x_{i}=g_{i}\left(u_{1}, \cdots, u_{p}\right), \quad i=1, \cdots, n,
$$

where the rank of the functional matrix

$$
\left(\begin{array}{c}
\partial g_{1} / \partial u_{1} \cdots \partial g_{1} / \partial u_{p} \\
\cdot \cdot \cdot \cdot \cdot \cdot \cdot \cdot \\
\partial g_{n} / \partial u_{1} \cdots \partial g_{n} / \partial u_{p}
\end{array}\right)
$$

is exactly $p$ and where the $g_{i}$ are analytic in the $u_{i}$ and defined for values of $u_{i}$ in an open convex $p$-cell, $P$, in the $E^{p}$ space of the $u_{i}$. Then the $p$ area, $A_{p}$, of this surface is given by

$$
A_{p}=\int \cdots \int\left\{\sum_{\left(l_{i}\right)} \frac{\partial\left(g_{l_{1}}, \cdots, g_{l_{p}}\right)^{2}}{\partial\left(u_{1}, \cdots, u_{p}\right)}\right\}^{1 / 2} d u_{1}, \cdots, d u_{p}
$$

If we use capital letters for the transform of the surface (3.2), the transformation being given by (3.1), its equation is, at "time $t$,"

$$
X_{i}=f_{i}\left(g_{1}, \cdots, g_{n}, t\right), \quad i=1, \cdots, n .
$$

Its area $A_{p}(t)$ is given by the integral

$$
A_{p}(t)=\int \cdots \int\left\{\sum_{\left(l_{i}\right)} \frac{\partial\left(X_{l_{1}}, \cdots, X_{l_{p}}\right)^{2}}{\partial\left(u_{1}, \cdots, u_{p}\right)}\right\}^{1 / 2} d u_{1} \cdots d u_{p}
$$

But by hypothesis $A_{p}(t) \equiv A_{p}$, and since this must be true for each piece of the surfaces we must have that the integrands in (3.5) and (3.3) are equal. Using the lemma of the last section this equality becomes 


$$
\begin{aligned}
& \sum_{\left(l_{i}\right)} \frac{\partial\left(g_{l_{1}}, \cdots, g_{l_{p}}\right)^{2}}{\partial\left(u_{1}, \cdots, u_{p}\right)}= \\
& \quad \sum_{\left(m_{i}\right)}\left\{\sum_{\left(n_{i}\right)} \frac{\partial\left(X_{m_{1}}, \cdots, X_{m_{p}}\right)}{\partial\left(x_{n_{1}}, \cdots, x_{n_{p}}\right)} \cdot \frac{\partial\left(g_{n_{1}}, \cdots, g_{n_{p}}\right)}{\partial\left(u_{1}, \cdots, u_{p}\right)}\right\}^{2} .
\end{aligned}
$$

We now proceed to draw consequences of (3.6) by making special choices of the $g_{i}$ of (3.2). For a particular choice we do two things: (a) verify that this reduction is true for $t=t_{0}$; (b) impose the condition that this be an identity in $t_{0}$, that is, see that these equations, after differentiation with respect to $t_{0}$, are verified at $t_{0}=t$.

(A) First choice of the $g_{i}$. Let

$$
\begin{array}{rlr}
x_{i} & =u_{i}, & i=1, \cdots, p, \\
x_{i} & =0, & i>p .
\end{array}
$$

We now verify (a) for (3.6) for this choice of the $g_{i}$. First,

$$
\frac{\partial\left(x_{l_{1}}, \cdots, x_{l_{p}}\right)}{\partial\left(u_{1}, \cdots, u_{p}\right)}=\left\{\begin{array}{l}
1, l_{i}=i, i=1,2, \cdots, p \\
0 \quad \text { otherisise. }
\end{array}\right.
$$

Therefore, (3.6) reduces to

$$
1=\sum_{\left(l_{i}\right)} \frac{\partial\left(X_{l_{1}}, \cdots, X_{l_{p}}\right)}{\partial\left(x_{1}, \cdots, x_{p}\right)} .
$$

Now at $t_{0}=t$

$$
\frac{\partial\left(X_{l_{1}}, \cdots, X_{l_{p}}\right)}{\partial\left(x_{1}, \cdots, x_{p}\right)}= \begin{cases}1 & \text { if } l_{i}=i, i=1, \cdots, p \\ 0 & \text { otherwise. }\end{cases}
$$

This last fact may be seen as follows: the expression $\partial X_{k} / \partial x_{j}$ is the derivative of the $k$ th component $X_{k}$, of a solution of (1.1) with respect to the $j$ th component of its initial point. According to (3.1)

$$
X_{i}=f_{i}\left(x_{10}, \cdots, x_{n 0}, t\right)
$$

and when $t=t_{0}, X_{i}\left(t_{0}\right)=f_{i}\left(x_{1}, \cdots, x_{n}, t_{0}\right)=x_{i}$. Thus $\partial X_{k} /\left.\partial x_{j}\right|_{t_{0}=t}=\delta_{k j}$, explaining the value of the last Jacobian. From this it follows that at $t_{0}=t(3.8)$, and hence (3.6), is verified.

We now verify (3.8) (that is, (3.6) reduced by this choice of the $g_{i}$ ) for condition (b). To do this we must differentiate (3.8) with respect to $t_{0}$ and then put $t_{0}=t$. The differentiation will produce a sum of products, and when $t_{0}$ is put equal to $t$ all products containing a factor of the form 


$$
\frac{\partial\left(X_{l_{1}}, \cdots, X_{l_{p}}\right)}{\partial\left(x_{1}, \cdots, x_{p}\right)},
$$

some $l_{j} \neq j$,

will be zero. Hence, eliminating all such terms before differentiation, (3.8) reduces on the right to one type of term, namely, those for which $l_{i}=i(i=1, \cdots, p)$

$$
0=\left.\sum_{i=1}^{p} \frac{\partial\left(X_{1}, \cdots, d X_{i} / d t_{0}, \cdots, X_{p}\right)}{\partial\left(x_{1}, \cdots, x_{p}\right)}\right|_{t_{0}=t} .
$$

And when $t_{0}=t, d X_{i} /\left.d t_{0}\right|_{t=t_{0}}=d X_{i} / d t=P_{i}\left(x_{1}, \cdots, x_{n}\right)=P_{i}$ so that condition (b) yields

$$
0=\sum_{i=1}^{p} \frac{\partial P_{i}}{\partial x_{i}} .
$$

If we now choose a set of $g_{i}$ as

$$
\begin{array}{rlr}
x_{1} & =0, \\
x_{i} & =u_{i}, & \\
x_{i} & =0, & i=2, \cdots, p+1, \\
& i>p+1,
\end{array}
$$

we shall, by the same route as above, arrive at the relationship

$$
\sum_{i=2}^{p+1} \frac{\partial P_{i}}{\partial x_{i}}=0 .
$$

This equation combined with (3.9) yields $\partial P_{1} / \partial x_{1}=\partial P_{p+1} / \partial x_{p+1}$ and in general we shall have (after suitable repetitions of this argument) that $\partial P_{i} / \partial x_{i}=\partial P_{k} / \partial x_{k}$. These combine with (3.9) to furnish the condition

$$
\partial P_{i} / \partial x_{i}=0,
$$

$$
i=1, \cdots, n \text {. }
$$

(B) Second choice of $g_{i}$. Put

$$
\begin{array}{rlr}
x_{i} & =u_{i}, \\
x_{p} & =x_{p+1}=u_{p}, & i=1, \cdots, p-1, \\
x_{i} & =0, & i>p+1 .
\end{array}
$$

We note first of all that

$$
\frac{\partial\left(x_{l_{1}}, \cdots, x_{l_{p}}\right)}{\partial\left(u_{1}, \cdots, u_{p}\right)}=\left\{\begin{array}{l}
1, l_{i}=i, i=1, \cdots, p-1, l_{p}=p \text { or } p+1, \\
0, \quad \text { otherwise. }
\end{array}\right.
$$

Therefore, if we use (3.11) and these last results, (3.6) reduces to 


$$
2=\sum_{\left(l_{i}\right)}\left\{\frac{\partial\left(X_{l_{1}}, \cdots, X_{l_{p}}\right)}{\partial\left(x_{1}, \cdots, x_{p}\right)}+\frac{\partial\left(X_{l_{1}}, \cdots, X_{l_{p}}\right)}{\partial\left(x_{1}, \cdots, x_{p-1}, x_{p+1}\right)}\right\}^{2}
$$

and this is easily seen to verify condition (a). Namely when $t_{0}=t$ the only nonzero terms are those for which

$$
\left(l_{1}, \cdots, l_{p}\right)=\left\{\begin{array}{l}
1,2, \cdots, p \\
1,2, \cdots, p-1, p+1 .
\end{array}\right.
$$

Proceeding as before, we verify (b) by differentiating (3.12)-and simplifying beforehand by dropping out all terms for which

$$
\left(l_{1}, \cdots, l_{p}\right) \neq\left\{\begin{array}{l}
1,2, \cdots, p, \\
1,2, \cdots, p-1, p+1,
\end{array}\right.
$$

since these will drop out when $t_{0}$ is put equal to $t$. This gives us

$$
\begin{aligned}
& 0=\left\{\frac{\partial\left(X_{1}, \cdots, X_{p}\right)}{\partial\left(x_{1}, \cdots, x_{p}\right)}+\frac{\partial\left(X_{1}, \cdots, X_{p}\right)}{\partial\left(x_{1}, \cdots, x_{p-1}, x_{p+1}\right)}\right\} \\
& \cdot \sum_{i=1}^{p}\left\{\frac{\partial\left(X_{1}, \cdots, d X_{i} / d t_{0}, \cdots, X_{p}\right)}{\partial\left(x_{1}, \cdots, x_{p}\right)}\right. \\
&\left.+\frac{\partial\left(X_{1}, \cdots, d X_{i} / d t_{0}, \cdots, X_{p}\right)}{\partial\left(x_{1}, \cdots, x_{p-1}, x_{p+1}\right)}\right\} \\
&+\left\{\frac{\partial\left(X_{1}, \cdots, X_{p-1}, X_{p+1}\right)}{\partial\left(x_{1}, \cdots, x_{p}\right)}+\frac{\partial\left(X_{1}, \cdots, X_{p-1}, X_{p+1}\right)}{\partial\left(x_{1}, \cdots, x_{p-1}, x_{p+1}\right)}\right\} \\
& \cdot \sum_{i=1}^{p+1}\left\{\frac{\partial\left(X_{1}, \cdots, d X_{i} / d t_{0}, \cdots, X_{p-1}, X_{p+1}\right)}{\partial\left(x_{1}, \cdots, x_{p}\right)}\right. \\
&\left.+\frac{\partial\left(X_{1}, \cdots, d X_{i} / d t_{0}, \cdots, X_{p-1}, X_{p}\right)}{\partial\left(x_{1}, \cdots, x_{p-1}, x_{p}\right)}\right\}
\end{aligned}
$$

where $\sum^{\prime}$ means the value $i=p$ is omitted. Putting $t=t_{0}$ reduces this to

$$
\begin{array}{r}
0=\sum_{i=1}^{p}\left\{\frac{\partial\left(X_{1}, \cdots, P_{i}, \cdots, X_{p}\right)}{\partial\left(x_{1}, \cdots, x_{p}\right)}+\frac{\partial\left(X_{1}, \cdots, P_{i}, \cdots, X_{p}\right)}{\partial\left(x_{1}, \cdots, x_{p-1}, x_{p+1}\right)}\right\} \\
+\sum_{i=1}^{p+1}\left\{\frac{\partial\left(X_{1}, \cdots, P_{i}, \cdots, X_{p-1}, X_{p+1}\right)}{\partial\left(x_{1}, \cdots, x_{p}\right)}\right. \\
\left.\quad+\frac{\partial\left(X_{1}, \cdots, P_{i}, \cdots, X_{p-1}, X_{p}\right)}{\partial\left(x_{1}, \cdots, x_{p-1}, x_{p+1}\right)}\right\} .
\end{array}
$$


The first term of the first brace is $\partial P_{i} / \partial x_{i}$ and the second term is zero except when $i=p$ when it is $\partial P_{p} / \partial x_{p+1}$; the first term of the second brace is zero except for $i=p+1$ when it is $\partial P_{p+1} / \partial x_{p}$ and the second term is $\partial P_{i} / \partial x_{i}$. Thus if we remove the $\partial P_{i} / \partial x_{i}$ which are zero, (3.13) gives us

$$
\frac{\partial P_{p}}{\partial x_{p+1}}+\frac{\partial P_{p+1}}{\partial x_{p}}=0
$$

A similar procedure based on corresponding choices of the $g_{i}$ produces

$$
\frac{\partial P_{k}}{\partial x_{l}}=-\frac{\partial P_{l}}{\partial x_{k}} \quad \text { for all } k, l
$$

(actually for $k \neq l$ but if we allow $k=l$ then (3.14) will include (3.10)).

From condition (3.14) we can derive very easily that the motion must be rigid, for differentiating (3.14) with respect to $x_{k}$ shows that

$$
\frac{\partial^{2} P_{l}}{\partial x_{k}^{2}}=-\frac{\partial}{\partial x_{k}} \cdot \frac{\partial P_{k}}{\partial x_{l}}=-\frac{\partial}{\partial x_{l}} \cdot \frac{\partial P_{k}}{\partial x_{k}} \equiv 0 .
$$

Thus the $P_{i}$ will be linear functions of the $x_{i}$ and by (3.14) skew symmetric. Equation (1.1) written in vector form is then

$$
\frac{d x}{d t}=A x+c
$$

where $x, d x / d t, c$ are column vectors and $A$ a skew-symmetric matrix $\left(A=\left(a_{i j}\right)\right.$ where $\left.a_{i j}=-a_{j i}\right)$. If one defines a new vector $z$ by the relationship $B z=x, B$ being nonsingular, then $z$ satisfies the vector differential equation

$$
\frac{d z}{d t}=B^{-1} A B \cdot z+B^{-1} c .
$$

And since $A$ is skew-symmetric we can find an orthogonal $B$ such that

$$
B^{-1} A B=\left(\begin{array}{cccc}
A_{1} & 0 & \cdots & 0 \\
0 & A_{2} & \cdots & 0 \\
\cdots & \cdots & \cdots & . \\
0 & \cdots & 0 & A_{k}
\end{array}\right) \text { or }\left(\begin{array}{cccc}
A_{1} & 0 & \cdots & 0 \\
0 & A_{2} & \cdots & 0 \\
\cdots & \cdots & . & . \\
0 & \cdots & A_{k} & 0 \\
0 & \cdots & 0 & 0
\end{array}\right) \text {, }
$$

depending on whether $n$ is even or odd and where 


$$
A_{i}=\left(\begin{array}{cc}
0 & \alpha_{i} \\
-\alpha_{i} & 0
\end{array}\right)
$$

where $\alpha_{i}$ is a constant (and real). The variables thus separate and the equations of the components of $z$ can be integrated in pairs directly (trivially if $A_{i}=(0)$. For example, if $\alpha \neq 0$

$$
\frac{d x_{1}}{d t}=\alpha x_{2}+c_{1}, \quad \frac{d x_{2}}{d t}=-\alpha x_{1}+c_{2}
$$

when integrated will give a rotation of angular velocity $\alpha$ about the point $\left(-c_{2} / \alpha, c_{1} / \alpha\right)$.

4. Proof of Theorem II. Let the correspondence $y_{i}=f_{i}\left(x_{1}, x_{2}, x_{3}\right)$ $(i=1,2,3), f_{i} \in C^{3}$, be an area-preserving map of $E^{3}$ onto itself. Since the surface $S: x_{i}=g_{i}\left(u_{1}, u_{2}\right)(i=1,2,3)\left(g_{i} \in C^{3},\left(u_{1}, u_{2}\right) \in R, R\right.$ a convex, open two-cell) and its image under this map have equal areas we must have

$$
\begin{aligned}
\iint_{R}\left\{\sum_{i} \frac{\partial\left(g_{i} g_{i+1}\right)^{2}}{\partial\left(u_{1}, u_{2}\right)}\right\}^{1 / 2} d u_{1} d u_{2} \\
=\iint_{R}\left\{\sum \frac{\partial\left(f_{i}\left(g_{r}\right) f_{i+1}\left(g_{8}\right)\right)^{2}}{\partial\left(u_{1}, u_{2}\right)}\right\}^{1 / 2} d u_{1} d u_{2} .
\end{aligned}
$$

Moreover the integrands must be equal, hence, by the lemma of $\$ 2$,

$$
\sum_{i=1}^{3} \frac{\partial\left(g_{i}, g_{i+1}\right)^{2}}{\partial\left(u_{1}, u_{2}\right)}=\sum_{j=1}^{3}\left(\sum_{k=1}^{3} \frac{\partial\left(f_{j}, f_{j+1}\right)}{\partial\left(x_{k}, x_{k+1}\right)} \frac{\partial\left(g_{k}, g_{k+1}\right)}{\partial\left(u_{1}, u_{2}\right)}\right)^{2} .
$$

This expression must be an identity in the $g_{i}$. Choosing $g_{1}=u_{1}$, $g_{2}=u_{2}$ and $g_{3}=$ const. (and making two similar choices) yields

$$
1=\sum_{i=1}^{3} \frac{\partial\left(f_{i}, f_{i+1}\right)^{2}}{\partial\left(x_{j}, x_{j+1}\right)}, \quad j=1,2,3 ;
$$

choosing $g_{1}=u_{1}, g_{2}=g_{3}=u_{2}$ (and making two similar choices) yields

$$
2=\sum_{i=1}^{3}\left(\frac{\partial\left(f_{i}, f_{i+1}\right)}{\partial\left(x_{j}, x_{j+1}\right)}+\frac{\partial\left(f_{i}, f_{i+1}\right)}{\partial\left(x_{j+2}, x_{j+3}\right)}\right)^{2}, \quad j=1,2,3 .
$$

Combination of these last two restrictions yields

$$
0=\sum_{i=1}^{3} \frac{\partial\left(f_{i}, f_{i+1}\right)}{\partial\left(x_{j}, x_{j+1}\right)} \frac{\partial\left(f_{i}, f_{i+1}\right)}{\partial\left(x_{j+2}, x_{j+3}\right)}, \quad j=1,2,3 .
$$

The first and last of these relations tell us immediately that the 3 
vectors $A_{i}=\left(\partial\left(f_{1}, f_{2}\right) / \partial\left(x_{i}, x_{i+1}\right), \partial\left(f_{2}, f_{3}\right) / \partial\left(x_{i}, x_{i+1}\right), \partial\left(f_{3}, f_{1}\right) / \partial\left(x_{i}, x_{i+1}\right)\right)$ are normal and orthogonal. $A_{i}$ represents the normal to a surface element which is the image of a plane element perpendicular to the $(i+2)$ th coordinate axis. Thus we know that the families of planes parallel to the coordinate planes must be carried into a triply orthogonal family of surfaces. Thus excluding the rigid motions it follows from the proof of Liouville's theorem on three-dimensional conformal maps ${ }^{2}$ that the map must be an inversion. But such maps do not preserve areas of spheres concentric to center of inversion and so are excluded-leaving the rigid motions (and reflections, of course).

University of California at Berkeley

${ }^{2}$ Blaschke, Differentialgeometrie, 3d ed., Berlin, Springer, p. 100. 